引用格式: 王勇, 韩舒婉, 李嘉源, 等. 五大交通运输方式碳达峰的经验分解与情景预测: 以东北三省为例 [J]. 资源科学, 2019, 41(10): 1824-1836. [Wang Y, Han S W, Li J Y, et al. Empirical decomposition and peak forecast of carbon emissions in five major transportation modes: Taking the three provinces in Northeast China as examples[J]. Resources Science, 2019, 41(10): 1824-1836.] DOI: $10.18402 /$ resci.2019.10.06

\title{
五大交通运输方式碳达峰的经验分解与情景预测 以东北三省为例
}

\author{
王 勇 ${ }^{1,2}$, 韩舒婉 ${ }^{1}$, 李嘉源 ${ }^{3}$, 李 博 ${ }^{1}$ \\ (1. 东北财经大学统计学院, 大连 116025 ; \\ 2. 东北财经大学博士后科研流动站, 大连 116025 ; \\ 3. 大连海事大学轮机工程学院, 大连 116026)
}

\begin{abstract}
摘 要: 交通业是国民经济发展和居民生活必需的基础产业之一, 也是碳排放的主要来源之一。高能耗、高污 染一直都是交通业的问题, 有效控制交通业碳排放量, 对于实现中国的碳排放达峰目标具有重要意义。本文以中 国东北三省为研究区,对公路、铁路、航空、水路和管道 5 种不同交通运输方式的碳排放进行了细分研究。首先,使 用广义迪氏指数 (GDIM) 模型分别考察了 2005-2016年 5 种交通运输方式碳排放的影响因素,在此基础上使用蒙 特卡洛模拟对 2017-2030年的五大交通运输方式碳排放的年平均变化率进行动态情景分析。结果显示: 投资规模 是影响铁路、公路、航空及管道运输碳排放量的首要因素,运输规模是影响水路运输的碳排放量的首要因素; 在同 一时间段内,各影响因素对不同类型运输方式碳排放的作用并非完全相同; 不同时间段内,同一影响因素对碳排放 的促增效应与促降效应也不同; 除基准情景外,2017-2030 年5种运输方式的碳排放量均逐渐下降; 技术突破情景 下, 5 种运输方式碳排放量预期下降幅度最大。研发使用清洁能源的运输设备、提高其使用性能并进行大力推广等 应当作为未来交通业节能减排的主要发展路径。
\end{abstract}

关键词: 交通业; 碳达峰; 经验分解; 情景预测 ; 蒙特卡洛模拟; 东北三省

DOI :10.18402/resci.2019.10.06

\section{1 引言}

减少温室气体排放已经成为全球性共识。国 际组织 “全球碳计划” (简称 GCP) 发表的研究报告 《2017年全球碳预算》 ${ }^{[1]}$ 显示, 与 2016 年相比, 2017 年底全球化石燃料燃烧和工业活动产生的 $\mathrm{CO}_{2}$ 排放 量上升了约 $2 \%$, 中国的碳排放增长了 $3.5 \%$ 。中国 作为世界上最大的碳排放国家, 碳排放量占全球总 量的 $28 \%$ 。为积极承担节能减排的国际责任, 2014 年 11 月 12 日, 中国向国际社会作出承诺: 2030 年左
右 $\mathrm{CO}_{2}$ 排放达到峰值且将努力早日达峰, 非化石能 源占一次能源消费比重提高到 $20 \%$ 左右。中国承 诺碳排放达峰给国内能源结构、产业结构调整带来 巨大转型压力和挑战,包括经济、能源和技术上的 协同和权衡, 因此, 中国能否实现 2030 年碳排放达 峰存在很大的不确定性及现实压力。

作为国民经济发展和居民生活必需的基础产 业之一, 交通业在运输服务中需要消耗大量的能 源, 特别是产生高碳排放量的化石燃料。IPCC 报告 ${ }^{1}$

收稿日期: 2019-04-22, 修订日期: 2019-07-30

基金项目: 教育部人文社会科学研究青年项目(18YJC910013); 辽宁省财政科研基金项目(重点课题)(18B010); 辽宁省教育厅科研项目 (LN2019Q48); 国家社会科学基金项目(19FTJB004)。

作者简介:王勇,男,山东临沂人,博士,副教授,硕士生导师,从事资源环境统计分析。E-mail: ywang@dufe.edu.cn

通讯作者: 韩舒婉,女,吉林白山人, 硕士研究生, 研究方向为资源环境统计分析。E-mail: shuwanhan@163.com

(1) 《2006年IPCC 国家温室气体清单指南》(政府间气候变化专门委员会2006 编写)。 
察多个绝对量的影响, 分解结果不受恒等式的约 仅次于能源供应部门和工业生产部门。2013 年,世 界自然基金会发布的报告 ${ }^{[2]}$ 显示, 交通业的平均碳 排放强度约为金融行业的 119 倍。所以, 研究交通 业的能源消耗特点,有效控制交通业的碳排放量, 对于实现中国的低碳发展目标具有重要意义。近 年来, 随着东北地区城市化进程的不断加快, 交通 业发展迅猛。总体来看, 东北地区的交通运输系统 发展较早、较为完善, 包括铁路、公路、水路、航空及 管道运输 5 种运输方式, 是中国交通运输方式的缩 影。对东北三省交通业的碳排放总量进行控制, 识 别影响东北地区交通业碳排放量变化的因素, 并预 测不同交通运输方式碳排放的未来变化, 对东北地 区未来碳排放政策的制定具有重要的现实意义, 对 中国其他地区交通业减排政策的制定也具有借鉴 作用。

\section{2 文献综述}

对碳排放进行研究时, 影响因素和达峰时间预 测是目前学术界的研究热点。这些研究主要可以 分为两大类。

第一类是对碳排放进行因素分解, 确定影响碳 排放的主要因素, 在此基础上, 对碳排放达峰时间 进行预测。广泛使用的方法有对数平均迪氏指数 (LMDI) 分解法 ${ }^{[3-5]}$, 回归模型 ${ }^{[6]}$, Kaya 恒等式 ${ }^{[7]}$, STIRPAT 模型 ${ }^{[8-10]}$ 等。其中, Kaya 恒等式及 LMDI 分 解法对碳排放进行分解, 但两种方法均受到恒等式 或分解过程中其他因素的约束, 使得因素之间存在 一定的依赖性无法考察每个影响因素的弹性 ${ }^{[1]}$ 。此 外, 指数分解法最多只能考察一个绝对量因素对碳 排放量产生的影响 ${ }^{[12]}$, 忽略了隐含的其他绝对量变 量, 造成分析结果具有片面性。在影响因素的选取 方面, 常见思路有两种, 一种是从宏观层面选取影 响因素, 如人均 $\mathrm{GDP}^{[3]}$ 、城镇化水平 ${ }^{[8]}$ 、人口规模及气 候差异 ${ }^{[10]}$ 等; 另一种从产业层面选取, 如产业结构 ${ }^{[7,10]}$ 、 产业增加值或产业占比 ${ }^{[3,10]}$ 。现有交通业碳排放研 究在进行因素分解时选取的影响因素并不全面, 均 忽视了投资因素对交通业碳排放的影响。因此本 文首次采用GDIM模型对交通业碳排放进行因素分 解。GDIM模型不仅计算简便, 结果准确, 还可以考

束, 消除了变量之间的依赖性, 不仅能克服已有指 数分解模型的缺点, 还能研究潜在影响因素对碳排 放量的影响, 且分解结果对不同因素之间的关联性 做了区分, 解决了因素之间重复计算的问题, 能够 更加准确全面地分析各因素对交通行业的碳排放 量的变动的实际影响。在变量选择和数据选取方 面, 引人了投资规模、投资效率与投资强度 3 个投资 相关因素, 考察投资对交通行业碳排放演变的 影响。

第二类是直接建立模型对碳排放量进行预 测。此类研究中常用的方法有 LEAP 模型 ${ }^{[13]} 、$ BP 神 经网络预测模型 ${ }^{[14]}$ 。传统计量模型 ${ }^{[8]}$ 对碳排放峰值 进行预测, 但碳排放的影响是复杂的非线性系统, 传统计量模型会受到模型及变量的选择和参数估 计等影响造成严重误差。LEAP模型的计算过程繁 琐且涉及到许多技术参数的设置, 缺少官方公布的 数据进行可靠支持, 导致研究结果误差较大。此 外, 情景分析法也是一种常见的辅助预测模型, 该 模型能够通过设置不同的情景, 辅助预测最优达峰 路径, 确定最优碳减排方法。但该方法无法单独使 用, 常常以“方法 X+情景分析法”的形式出现, 其中 常见的方法主要有环境库兹涅茨曲线 ${ }^{[6]}$ 、STIRPAT 模型 ${ }^{[9]} 、$ LEAP 模型 ${ }^{[13]} 、$ BP 神经网络预测模型 ${ }^{[14]} 、$ 自下

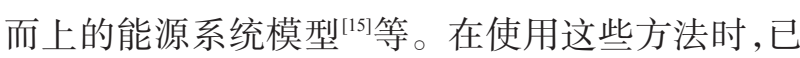
有研究大多将各影响因素设为固定的变化率, 忽略 了未来的风险和不确定性。因此使用蒙特卡洛模 拟对交通业碳排放的未来趋势进行预测,该方法可 以考虑不确定性的条件下根据各相关因素的历史 演变情况对变量未来的趋势给出可能性最大的演 化路径, 其预测结果更加科学合理。

从交通业碳排放的已有研究来看, 既有针对整 体交通业碳排放的研究 ${ }^{[6,15]}$, 也有针对某一类具体交 通运输方式碳排放的研究, 如公路运输 ${ }^{[8,13]}$, 还有针 对多个细分交通运输方式碳排放的研究 ${ }^{[3]}$ 。从研究 内容来看, 影响因素是已有交通业碳排放研究的主 要内容 ${ }^{[3,815]}$, 对交通业碳排放的预测分析也得到越 来越多的关注 ${ }^{[13,15]}$ 。已有研究为本文奠定了良好的 研究基础, 同时,已有研究在模型构建、变量选取、 
情景设计、交通业细分分类等方面的不足也是本文 研究的出发点。

基于已有研究现状, 本文考虑到投资规模、投 资效率与投资碳强度 3 个投资相关因素对 5 种交通 运输方式的影响, 运用 GDIM 模型分别对东北三省 5 种交通运输方式进行因素分解, 并结合国家相关 政策目标设定 5 种交通运输方式的各影响因素的潜 在年均变化率,运用蒙特卡洛模拟进行情景预测。

\section{3 模型构建}

\section{1 交通业碳排放因素分解的模型构建}

本文采用 Vaninsky ${ }^{[15]}$ 提出的 GDIM 分解法对东 北地区交通业碳排放的影响因素进行分解。GDIM 基于变形的 Kaya 恒等式,构建包括多个绝对因素和 相对因素的多维因素分解模型, 能够揭示交通业碳 排放的影响因素。本文选择能源消费总量、总服务 量及固定资产投资总额 3 个绝对量因素作为碳排放 的影响因素,其余变量均由公式分解得到。

基于 GDIM, 建立交通业碳排放影响因素的表 达式, 如式(1)-(3):

$$
\begin{gathered}
C=Y \times(C / Y)=E \times(C / E)=I \times(C / I) \\
E / Y=(C / Y) /(C / E) \\
Y / I=(C / I) /(C / Y)
\end{gathered}
$$

式中: $C$ 为东北三省交通业各运输方式的碳排放总 量; $E$ 为各运输方式的能源消费总量; $I$ 为各运输 方式的固定资产投资总额,包括扩建、改建、迁建及 恢复等; $C / E$ 为能源消费碳强度, 即消耗每吨标准 煤能源释放的碳排放量; $C / I$ 为投资碳强度, 即每 单位投资金额释放的碳排放量; $Y$ 为交通业的总服 务量 (即换算周转量); $C / Y$ 为运输碳强度, 即每单 位周转量释放的碳排放量; $E / Y$ 为能源强度, 即每 单位周转量消耗的能源总量; $Y / I$ 为投资效率, 即每 单位投资金额可以周转的总成果。

由于货物与旅客周转量无法直接相加, 因此引 人换算周转量的概念, 具体公式如下:

换算周转量 $=$ 货物周转量 +

(旅客周转量 $\times$ 客货换算系数 $)$

根据已有资料 ${ }^{[5,16,17]}$, 中国交通运输客货换算系 数为铁路为 1.00 、公路为 0.10 、水路为 0.33 及航空为 0.07 , 单位为 $\mathrm{t} \cdot \mathrm{km} /($ 人 $\cdot \mathrm{km})$ 。
进而, 可以将(1)-(3)变换为式(5)-(9):

$$
\begin{gathered}
C=Y \times(C / Y) \\
Y \times(C / Y)-E \times(C / E)=0 \\
Y \times(C / Y)-I \times(C / I)=0 \\
Y-I \times(Y / I)=0 \\
E-Y \times(E / Y)=0
\end{gathered}
$$

交通业的碳排放变化可以被分解为 8 种影响因 素之和: $\Delta Y 、 \Delta E 、 \Delta I 、 \Delta C / Y 、 \Delta C / E 、 \Delta Y / I 、 \Delta E / Y$ 以及 $\Delta C / I$ 。其中, 3 个绝对量因素为 $\Delta Y 、 \Delta E$ 、 $\Delta I$, 分别表示交通业运输规模的变化对碳排放的影 响、能源消耗规模的变化对碳排放的影响及投资规 模变化对碳排放的影响。在相对量因素中, $\Delta C / Y$ 表示交通运输方式的低碳程度, $\Delta C / E$ 与 $\Delta C / I$ 分别 表示能源消费的低碳程度与投资的低碳程度的变 化与调整,即能源消费的结构变化对交通业碳排放 变化的影响, $\Delta Y / I$ 表示交通业成本的变化对碳排放 变化的影响, $\Delta E / Y$ 表示各运输方式的单位周转量 消耗的能源总量的变化对碳排放的影响。

\section{2 碳排放的核算方法及数据来源}

本文参考《中国省级温室气体清单编制指南》 ${ }^{[18]}$ 公布的碳排放计算方法和参数,并结合东北三省统 计局官方公布的相关参数, 从化石燃料的消耗角度 对东北地区交通行业的碳排放量进行估算,具体公 式如下:

$$
C=\sum_{i=1}^{11} \sum_{j=1}^{5} E_{i j} \times C V_{i j} \times C C F_{i j} \times C O F_{i j} \times(44 / 12)(10)
$$

式中: $C$ 表示交通业的碳排放总量, 单位为万 $\mathrm{t}$; $i=1,2, \cdots, 11$ 表示能源种类, 本文根据 IPCC 的能源 划分及交通业燃料的特殊性选取了原煤、洗精煤、 焦炭、原油、汽油、煤油、柴油、燃料油、液化石油气、 炼厂干气及天然气 11 种能源; $j=1,2, \cdots, 5$ 表示铁 路、公路、水路、航空及管道运输 5 种运输方式; $E$ 为 终端化石能源消费总量, 单位为万 $\mathrm{t}$ 标准煤; $C V$ 为 平均低位发热值, 单位为 $\mathrm{kJ} / \mathrm{kg}$ 或 $\mathrm{kJ} / \mathrm{m}^{3} ; C C F$ 为能 源的碳含量, 单位为 $\mathrm{kg} / 10^{6} \mathrm{~kJ} ; C O F$ 为能源的碳氧 化率; 44/12 表示 $\mathrm{CO}_{2}$ 与碳的分子量之比。

本文选取 2005-2016年东北地区交通业的数 据为样本区间, 各变量的数据分为 5 个交通运输方 式即铁路、公路、水路、航空及管道运输。国家统计 
局官方仅对运营的水路运输及航空运输进行统计, 铁路与管道运输不含私人部门。因此, 在对上述 4 个部门进行研究时, 仅对其运营部门进行研究与分 析。公路运输则以估算的私家车与运营车辆为研 究对象。

使用的数据来源于 2005-2017的《辽宁统计年 鉴》 ${ }^{[19]}$ 、《吉林统计年鉴》 ${ }^{[20]}$ 及《黑龙江统计年鉴》 ${ }^{[21]}$, 各能源的换算系数及平均低位发热值来源于《中国 能源统计年鉴 (2014) 》 ${ }^{[22]}$, 碳含量和碳氧化率数据来 源于《中国省级温室气体清单编制指南》 ${ }^{[18]}$ 。为了保 证数据的可比性,剔除价格因素对变量产生的影 响,本文将交通业的固定资产投资的金额以 2005 年 为不变价进行平减。

\section{3 交通业情景预测模型构建}

由下文中因素分解结果可知, 在东北三省的交 通业碳排放的演变过程中, 不同种类的交通运输方 式影响因素不完全一致。因此, 本文在进行情景预 测时分别对 5 种运输方式进行预测。由分解结果可 知, 铁路、公路、航空及管道运输的主要促增因素为 投资规模, 而运输碳强度、投资碳强度和投资效率 的下降会有力地促进碳排放的下降, 因此未来具有 很大的减排潜力。水路运输的主要促增因素为总 服务量及能源消费碳强度, 运输碳强度和能源强度 具有很大的减排潜力。减排潜力大的因素是交通 业制定减排政策时着重考虑的主要方面。情景分 析中,设碳排放量、投资规模、总服务量、投资碳强 度、投资效率、运输碳强度、能源强度、能源消费碳 强度的变化率分别为 $c 、 i 、 y 、 a 、 b 、 d 、 e 、 f$, 由 于各运输方式的促增因素及具有很大减排潜力的 因素不完全相同, 因此对铁路、公路、航空及管道运 输构造式(11), 对水路运输构造式(12):

$$
\begin{aligned}
C_{t+1}= & I_{t} \times(1+i) \times(C / I)_{t} \times(1+a) \times(Y / I)_{t} \\
& \times(1+b) \times(C / Y)_{t} \times(1+d) \\
C_{t+1}= & Y_{t} \times(1+y) \times(C / Y)_{t} \times(1+d) \times(E / Y)_{t} \\
& \times(1+e) \times(C / E)_{t} \times(1+f)
\end{aligned}
$$

因此,碳排放量 $(C)$ 的变化率为式 $(13) 、(14)$ :

$$
\begin{aligned}
& c=(1+i) \times(1+a) \times(1+b) \times(1+c)-1 \\
& c=(1+y) \times(1+c) \times(1+d) \times(1+e)-1
\end{aligned}
$$

\section{4 交通业碳排放预测的情景设定}

本文基于交通业各影响因素过去的变化趋势、 减排潜力及减排政策实施的难易度,将未来发展情 景设定为基准情景、低碳情景及技术突破情景 3 种。

(1)基准情景。基准情景是以交通业过往的发 展特征为基础,假设当下的经济与技术环境不变, 政府不出台新的减排措施, 交通部门的发展及各项 相关指标的发展采用趋势外推。为了全面地反映 不同运输方式的影响因素的惯性发展趋势及减排 潜力, 本文参考 $\operatorname{Lin}^{[23]}$ 对基准情景的分析, 设定东北 地区交通业相关因素的潜在变化幅度,计算出 2005-2016 年、2009-2016 年及 2013-2016 年各 因素的年平均变化率, 2017-2030年各因素的潜在 年平均变化率的最小值、中间值及最大值分别对应 上述 3 个时期中的最小值、中间值及最大值。基准 情景下各因素的潜在变化率见表 1 。

(2)低碳情景。在低碳情景下,政府大力提高 能源使用效率, 优化交通业能源消费结构,投资效 率有所增强,投资规模进人平稳的中速阶段。由于 文章篇幅所限, 此处仅列举参考依据, 设置过程不 具体说明, 低碳情景下各因素的变化率设置见表 1 。

总服务量的潜在变化率的中间值的设置参考 当下中国人口数量计算人口增长率, 交通运输碳强 度的设置参考《“十三五”现代综合交通运输体系发 展规划》 ${ }^{[24]}$ 及邢丽敏 ${ }^{[25]}$ 的研究结果。参考当下东北 地区人口增长率, 将总服务量及运输碳强度指标的 潜在变化幅度设为 $0.2 \%$ 。能源强度的设置参考《能 源减排 “十三五”规划》 ${ }^{[26]}$ 及十三三五”现代综合交通 运输体系发展规划》 ${ }^{[24]}$, 能源消费碳强度则根据《能 源发展战略行动计划》 ${ }^{[27] 、}$ 、林伯强 ${ }^{[28]}$ 及《能源生产和 消费革命战略》|[29]进行设置。

投资相关指标方面, 投资规模参考中科院 ${ }^{[30]}$ 对 固定资产投资增速的预测, 考虑到铁路是未来国家 交通运输发展的主要方向及东北三省时中俄原油 管道二线的重要枢纽, 因此铁路运输与管道运输的 投资规模的变化率较其他运输方式偏高。由于前 文因素分解结果中显示, 公路运输与航空运输中, 运输规模与投资规模作用相似, 因此, 其投资规模 的变化率设置参考运输规模变化率的设置方式。

（3）技术突破情景。《交通运输节能环保“十三 


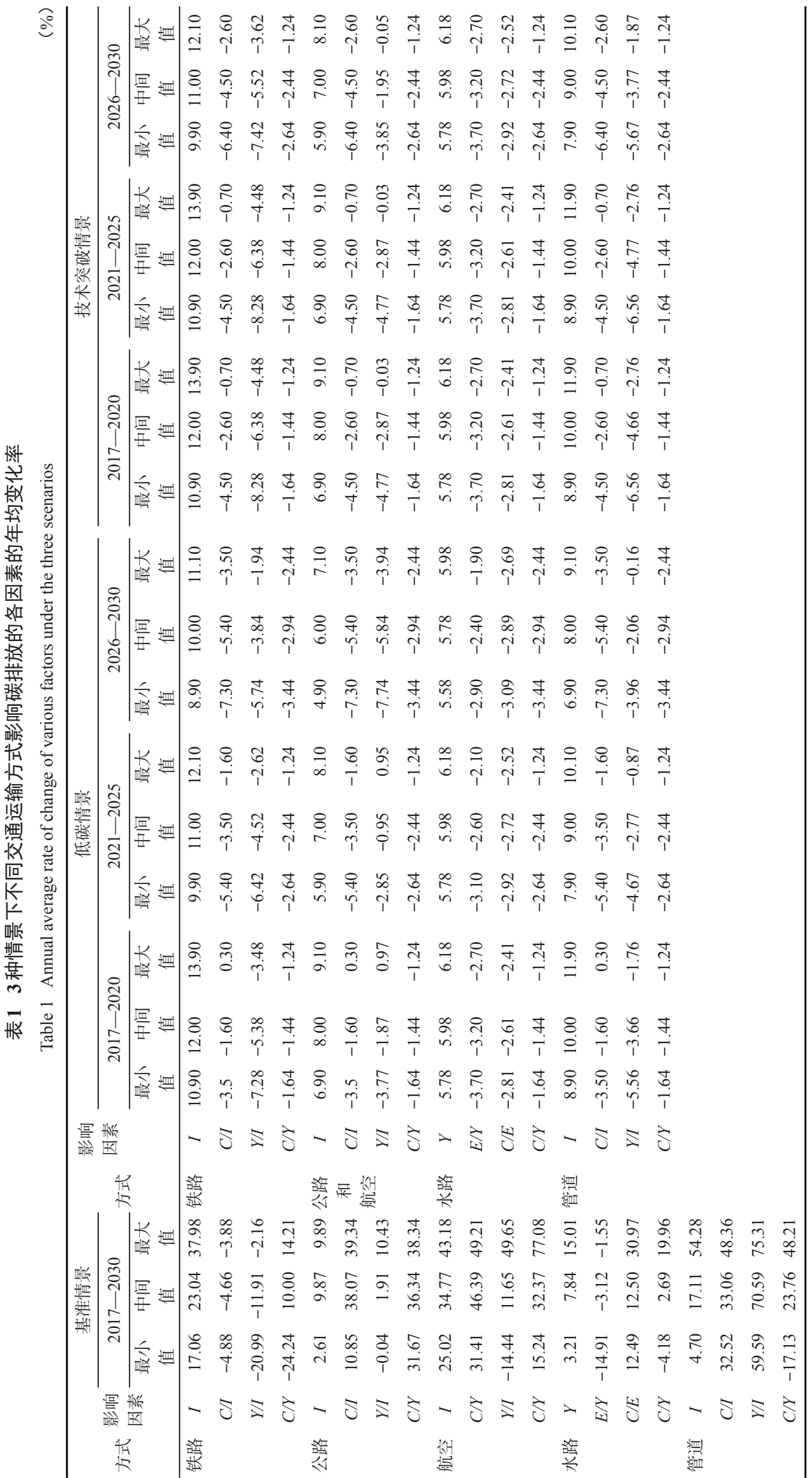


五” 规划》等1]中提出, 交通业要通过制度设计、技术进 步及结构调整, 加大科技研发力度, 促进资源节约 循环高效利用。本文在低碳情景的基础上,对影响 因素的潜在年平均变化率进行强化调整, 进一步得 到在能源技术实现突破的情景, 称之为技术突破情 景。与低碳情景相比,该情景将改变投资比例, 投 资于使用清洁能源的运输方式的更新与升级的比 例上升,而用于促进经济增长的扩大再生产的投资 比例相对减少。该情景的各因素变化率设置结果 见表 1 。

本文假定, 技术突破情景下, 投资规模的潜在 增长率保持不变, 用于扩大运输规模的投资相对减 少, 从而导致投资效率下降。参考 $\mathrm{Chen}^{[32]}$ 、《能源减 排“十三五”规划》登及及情景设置中 2021 年技术突破 效果开始发挥作用的假定,对投资效率进行设置。

运用蒙特卡洛模拟预测碳排放量的变化率, 若 已知变量最可能出现的结果及取值区间已知但概 率分布形状未知, 应当选择三角形分布 ${ }^{[33]}$ 。各影响 因素最可能出现的变化率为 3 种情景中的中间值, 结合三角形分布, 建立最大值、最小值与中间值的 概率分布关系。模拟时需给出变量的区间值, 因此 将各影响因素作为假设变量, 将变量在不同情景中 不同时间段内的中间值按大小作为该情景下的最 小值、中间值和最大值。由于在模拟过程中, 变量 可随机选取区间中的任意值, 因此结果更加贴近真 实值。本文使用Crystal Ball对基准情景、低碳情景 与技术突破情景中碳排放分别进行 50 万次模拟, 得 出最终结果。

\section{4 结果与分析}

\section{1 五大交通运输方式碳排放的因素分解结果}

由式 (7)、(10)可以计算得到东北三省 5 种交通 运输方式各影响因素的分解结果, 如图 1 所示。由 分解结果可知, 影响 5 种交通运输方式碳排放量的 因素不完全相同, 且同一影响因素对不同种类的运 输方式的促增或促减作用也不相同。

（1）2005-2016年, 交通业的运输规模与投资 规模对东北三省的 5 种交通运输方式几乎始终起促 增效应。其中运输规模的促增效应在公路运输、航 空运输和水路运输方面作用显著。在公路运输及
航空运输方面,运输规模的促增效应在 2005-2008 年、2009-2012 年均是引起碳排放量增加最多的因 素之一。但从总体趋势上看,运输规模所产生的促 增效应在逐渐减弱。对比之下,运输规模在水路运 输方面的促增效应在 3 个时间段内逐渐增加,产生 的碳排放量分别为 1300 万 $\mathrm{t} 、 2600$ 万 $\mathrm{t}$ 及 5200 万 $\mathrm{t}$ 。 投资规模的促增作用在铁路运输、公路运输、航空 运输及管道运输方面表现得格外明显。在铁路运 输方面,投资规模在 3 个时间段内均为主要促增因 素，投资规模产生的碳排放量分别达到 8900 万 t、7400 万 $\mathrm{t}$ 及 3900 万 $\mathrm{t}$ 。总体而言, 投资规模的促增效应在 铁路方面正逐渐减弱。2009-2012年,投资规模在 公路运输、管道运输方面产生的碳排放量分别高达 11400 万地 14600 万 $\mathrm{t}$ 。

（2）2005-2016年,投资碳强度、运输碳强度、 能源强度及能源消费规模对东北三省的 5 种交通运 输方式主要起促降效应。造成这一结果的主要原 因是国家层面的政策约束: “十一五”规划提出后, 中央及各地方政府更加重视节能减排，“十一五”规 划中首次提出能源强度约束指标, “十一五”和“十 二五” 期间能源强度的下调目标分别设定为 $20 \%$ 和 $16 \%{ }^{[34]}$ 。《能源减排 “十三五”规划》 ${ }^{[27]}$ 提出能源强度 在“十三五”期间下降 $15 \%$ 以上。因此,在未来一段 时间内, 能源强度的降低将可以继续促进东北三省 交通业的碳减排。

投资碳强度在公路运输、航空运输和水路运输 方面的促降作用显著。在公路运输方面,投资强度 在 2009-2012年促降效应达到最大值 22100 万 $\mathrm{t}$, 但 随后又变为促增效应, 促进碳排放量增长为 3900 万 $\mathrm{t}$ 。 航空运输方面,投资碳强度在 3 个时间段则始终起 促降效应,从 $2005-2008$ 年的 2600 万 $\mathrm{t}$ 变为 20092012 年的 5000 万 $\mathrm{t}$, 随后其促降效应又降至 2000 万 $\mathrm{t}$ 。 水路运输则与公路运输呈现相同的发展趋势, 即在 第三个时间段内再次反弹,变为促增因素,这说明 东北三省实施的节能减排措施力度有待进一步提 高。投资碳强度在铁路和管道运输上则存在一定 的滞后性,从第三个时间段即 “十一五”后期才开 始显现促降效应。

运输碳强度在公路运输与水路运输方面促降 效应显著。并将其修改为 2009-2012 年时间段到 

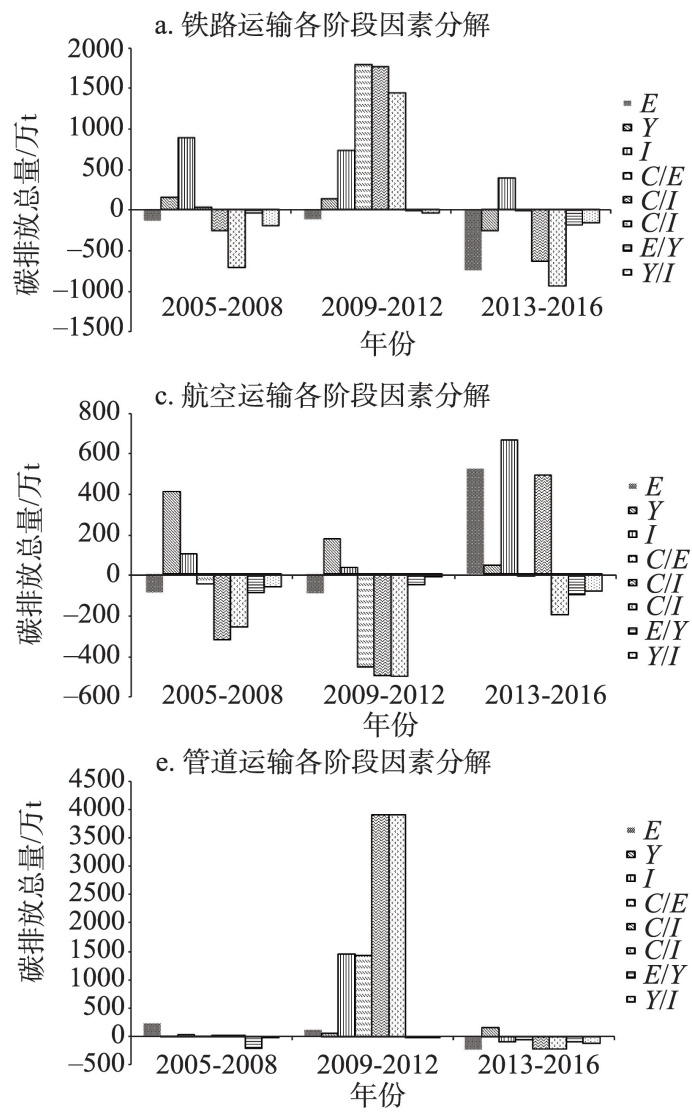

b. 公路运输各阶段因素分解

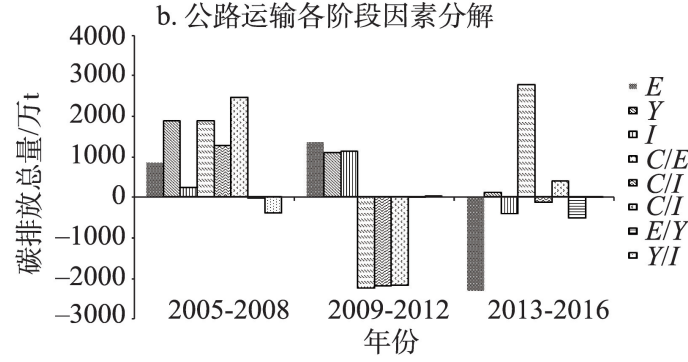

d. 水路运输各阶段因素分解

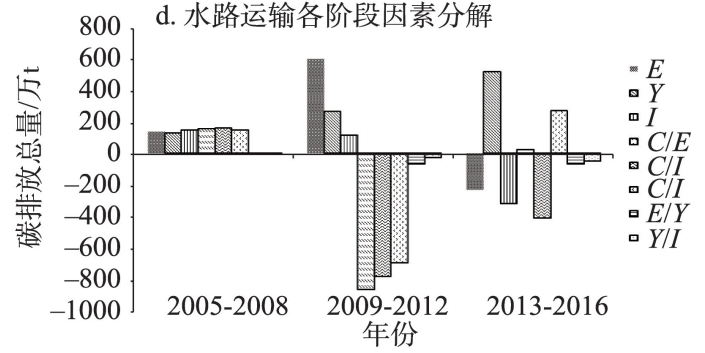

f. 各运输方式各阶段碳排放量变化图
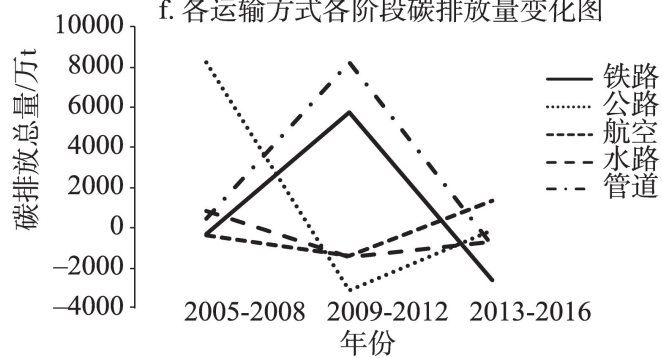

图 $12005-2016$ 年交通行业碳排放变化的分阶段因素分解结果

Figure 1 Staged factor decomposition results of carbon emissions changes in the transportation industry, 2005-2016

2013-2016年时间段, 公路运输与水路运输的运输 碳强度分别由 22100 万 $\mathrm{t}$ 下降至 1300 万 $\mathrm{t}$ 、由 7700 万 $\mathrm{t}$ 下降至 4100 万 $\mathrm{t}$ 。总体而言, 运输碳强度虽然在 2013-2016年呈现促降效应, 但下降幅度较小, 效 果较弱, 说明政策实施有待加强。在航空运输方 面, 2013-2016年反弹情况较为严重, 投资碳强度 变为促进碳排放最主要的三种因素之一。同样地, 铁路和管道运输上的滞后性仍旧存在, 均从第三个 时间段开始呈现促降效应。

能源强度在 5 种交通方式中, 始终起促降效 应。“十一五”规划后,其促降效应明显增强。

能源消费碳强度的变化较为复杂。能源消费 碳强度是影响公路运输碳排放的主要因素之一, 其 在第二个时间段内促降效应达到 22700 万 $\mathrm{t}$, 在第三 个时间段内促增效应达到 28000 万 $\mathrm{t}$, 即促进公路运 输产生 28000 万 $\mathrm{t}$ 的碳排放量。在航空运输与水路 运输方面, 能源消费碳强度在 2009-2012 年内促降 效应最大。而铁路与管道运输方面, 能源消费碳强
度在 2013-2016 年期间才开始逐渐显现出促降效 应, 且效应较小。

能源消费规模在铁路运输、公路运输及水路运 输方面促降效应明显。相较于前两个时间段, 在 2013-2016年期间,能源消费规模的促降效应分别 为 7600 万 $\mathrm{t} 、 23400$ 万 $\mathrm{t}$ 和 2300 万 $\mathrm{t}$, 有较大幅度的 提升。

\section{2 五大交通运输方式碳排放的达峰预测}

基于公式(13)-(14),使用蒙特卡洛模拟法对 东北地区交通业的碳排放的年均变化率进行随机 取值,预测 2017-2030年碳排放量的变化情况。图 2、图 3、图 4分别展示 3种情景下 2017-2030年东北 三省交通业 5 种交通运输方式的碳排放潜在年均增 长率。

(1)在基准情景下,公路运输、航空运输及管道 运输的未来预期碳排放量将至少变为现在的 1 2 倍,中国将无法实现 2030 年碳排放达峰的承诺。图 2 显示, 2017-2030 年东北三省交通业的 5 种交通 
2019年 10 月

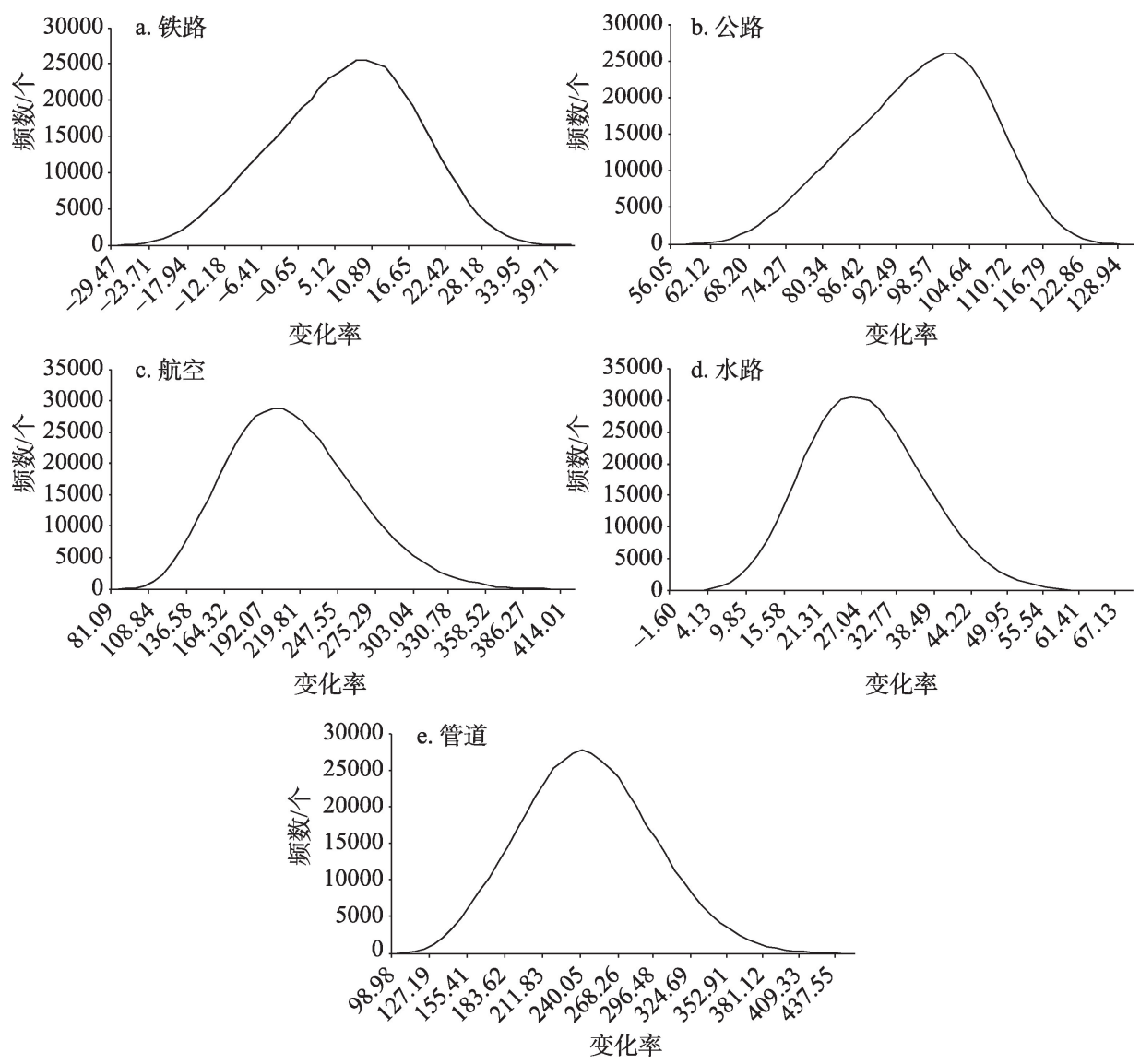

图 2 基准情景下 2017-2030 年东北三省五大交通运输方式碳排放的年均变化率的概率分布图

Figure 2 Probability distribution of annual average rate of change of carbon emissions from the five major transportation industries in the three northeastern provinces under the baseline scenario, 2017-2030
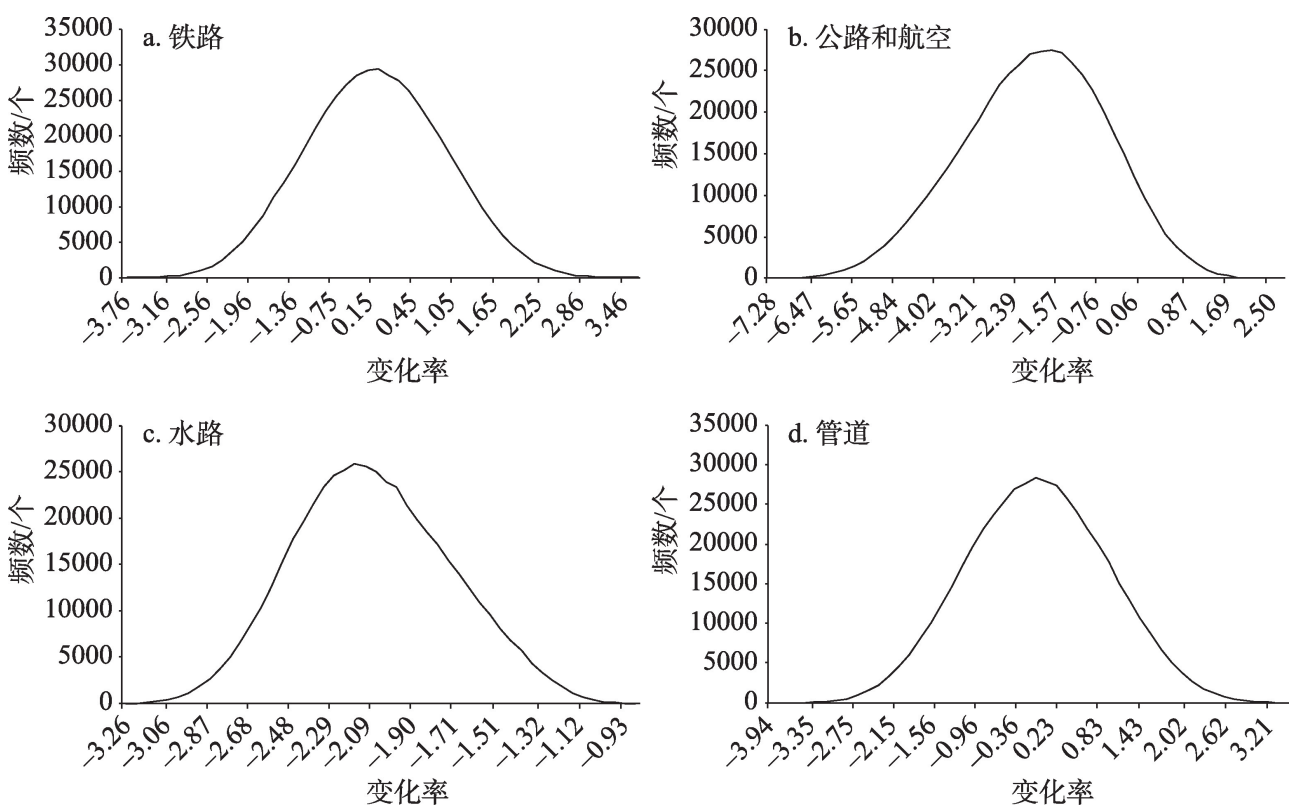

图 3 低碳情景下 $2017-2030$ 年东北三省五大交通运输方式碳排放的年均变化率的概率分布图

Figure 3 Probability distribution of annual average rate of change of carbon emissions from the five major transportation industries in the three northeastern provinces under the low-carbon scenario, 2017-2030 

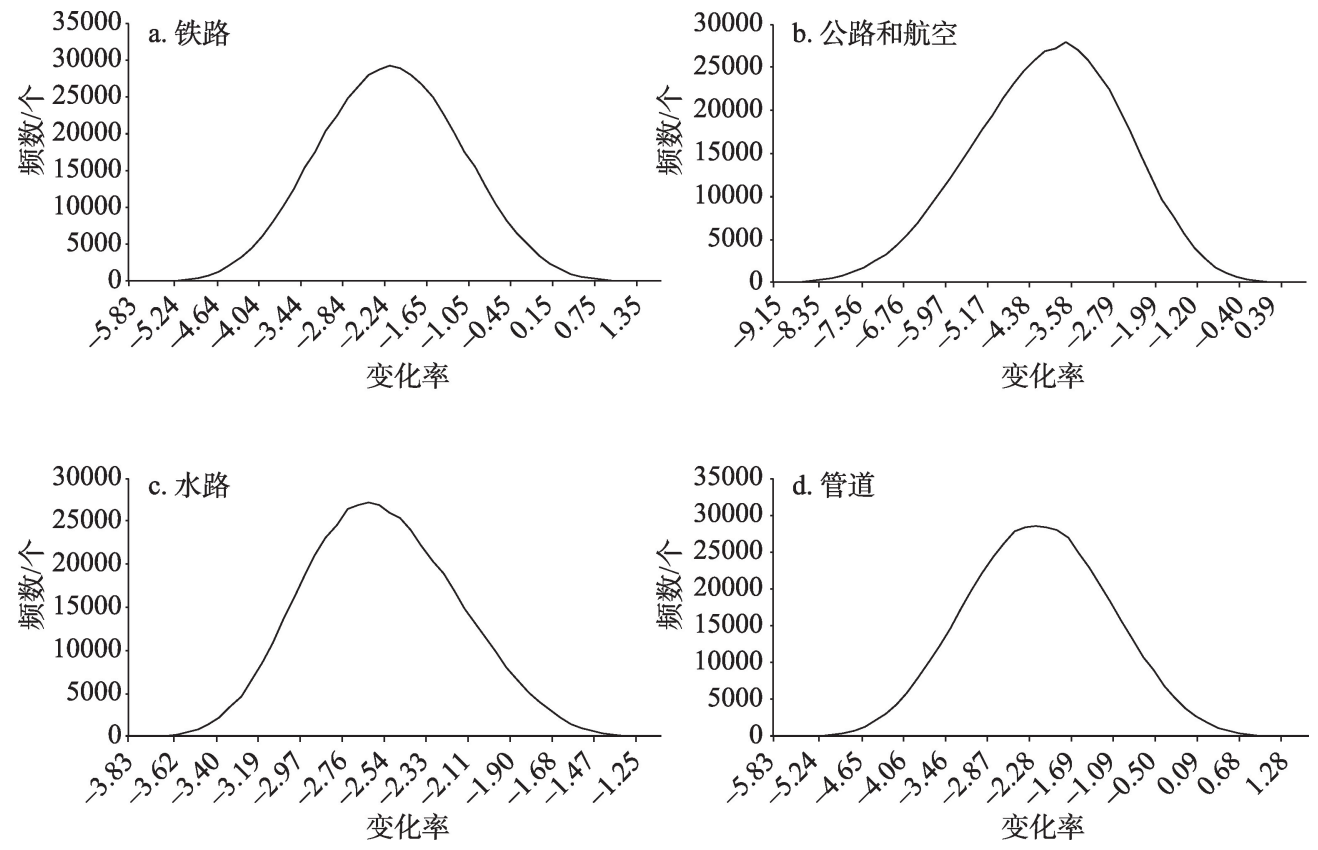

图 4 技术突破情景下 2017-2030 年东北三省五大交通运输方式碳排放的年均变化率的概率分布图

Figure 4 Probability distribution of annual average rate of change of carbon emissions from the five major transportation industries in the three northeastern provinces under the technological breakthrough scenario, 2017-2030

运输方式碳排放的年增长率可能持续增长, 且增长 幅度较大。公路运输、航空运输及管道运输碳排放 的年平均增长率将大概率出现在 100\% 104\%、 199\% 205\%及 $238 \%$ 244\%之间; 而增长幅度较小 的铁路运输与水路运输碳排放量的年均增长率在 $6 \% \sim 10 \%$ 和 $24 \% \sim 27 \%$ 之间的概率最大。

(2)在低碳情景下, 2017-2030年东北三省交 通业的 5 种交通运输方式碳排放的年增长率较基准 情景下有大幅度下降。图 3 显示, 铁路运输、公路及 航空运输、水路运输和管道运输碳排放的年平均增 长率将大概率出现在 $-0.05 \%$ $-0.30 \% 、-1.57 \%$ $-1.98 \%$ 、 $-2.15 \% \sim-2.25 \%$ 和 $-0.07 \% \sim-0.36 \%$ 之间。 这是由于该情景下各运输方式开始注重节能减排, 控制影响碳排放量增加的影响因素, 但由于各运输 方式的年平均增长率下降较小, 说明政府仍应继续 加大节能减排力度。

为了进一步比较该情景下各运输方式达峰时 间, 对各运输方式 2017-2020年、2021-2025 年及 2026-2030年分别进行蒙特卡洛预测, 其碳排放的 年均增长率的大概率范围见表 2 。

由表 3 可以看出, 水路运输是所有运输方式中 最先在 2017-2020年达峰的, 随后铁路运输在
2021-2025 年达峰, 公路及航空运输、管道运输在 2026-2030年达峰,但下降幅度较大。

（3）在技术突破情景下, 2017-2030 年东北三 省交通业的 5 种交通运输方式碳排放的年增长率较 基准情景和低碳情景下均有大幅度下降。图 4 显 示, 铁路运输、公路及航空运输、水路运输和管道运 输碳排放的年平均增长率出现在 $-2.10 \%$ - $2.30 \%$ 、 $-3.28 \% \sim-3.78 \%,-2.54 \% \sim-2.76 \%$ 和 $-2.10 \% \sim-2.30 \%$ 之间的可能性最大。因此, 技术突破情景是 3 种情 景中碳排放量下降幅度最大的,此时交通业的碳减 排也是 3 种情景中发展最好的。可以预见,低碳技 术的创新可以促使东北三省交通业完成能源的结 构调整与效率提升等重大目标, 推动其实现 2030 年 达峰目标。

同样地, 对五大运输方式在 2017-2020 年、 $2021-2025$ 年及 2026 - 2030 年年平均增长率分别 进行蒙特卡洛预测, 其大概率范围见表 3 。

由表 3 可以看出, 水路运输仍然是所有运输方 式中最先达峰的, 但其他 4 种运输方式均能在 2021-2025 年实现达峰目标。与低碳情景相比, 5 种运输方式在该情境下较低碳情景达峰时间均有 所提前,说明该情景减排效果更加明显。 
表 2 低碳情景下五大运输方式碳排放在各时间段 年平均增长率

Table 2 Annual average growth rate of each of the five major modes of transport under the low-carbon scenario

$(\%)$

\begin{tabular}{lccc}
\hline \multicolumn{1}{c}{ 运输方式 } & $2017-2020$ 年 & $2021-2025$ 年 & $2026-2030$ 年 \\
\hline 铁路 & $2.80 \sim 3.20$ & $-0.30 \sim-0.10$ & $-2.80 \sim-3.00$ \\
公路及航空 & $2.80 \sim 3.10$ & $0.00 \sim 0.01$ & $-8.20 \sim-8.50$ \\
水路 & $-1.47 \sim-1.51$ & $-1.86 \sim-1.91$ & $-2.65 \sim-2.70$ \\
管道 & $2.80 \sim 3.20$ & $0.20 \sim 0.50$ & $-2.70 \sim-3.10$ \\
\hline
\end{tabular}

\section{5 结论与建议}

\section{1 结论}

本文首先使用 GDIM 模型对 2005-2016年东 北三省交通业的 5 种交通运输方式的碳排放量分别 进行因素分解,在此基础上结合政府制定的相关政 策目标设置各运输方式的主要影响因素的预期年 平均变化率,再使用蒙特卡洛模拟对 2017-2030年 的年平均变化率进行动态情景分析。主要研究结 论如下:

(1) 投资规模是影响铁路运输、公路运输、航空 运输及管道运输碳排放量的首要因素, 运输规模是 影响水路运输的碳排放量的首要因素。在同一时 间段内, 对于不同种类的运输方式, 各影响因素的 作用不完全相同。如 2013-2016年, 能源消费碳强 度是公路运输最主要的促增因素, 但对铁路运输、 航空运输及管道运输却有相反作用。对于同一种 运输方式而言,不同时间段内同一影响因素的影响 方向不同。以铁路运输为例, 运输碳强度与投资碳 强度在 2005-2008 年、2013-2016 年均起促降效 应,在 2009-2012年内起促增效应。

(2)不同的情景下 5 种交通运输方式的碳排放 量的潜在变化率存在明显差异。基准情景下, 5 种 运输方式的碳排放量将会继续上升,截至 2030 年, 公路运输的碳排放量较 2016 年碳排放量翻一番,航 空运输与管道运输的碳排放量较 2016年甚至会翻 两番。除基准情景外, 2017-2030年 5 种运输方式 的碳排放量都是逐渐下降的,均能够实现 2030 年交 通业达峰的目标, 但相比较而言, 技术突破情景下 5 种运输方式碳排放量的年均下降水平较高, 碳减排 效果较好。

\section{表3 技术突破情景下五大运输方式碳排放在各时间段 年平均增长率}

Table 3 Annual average growth rate of each of the five major modes of transport in the context of technological breakthroughs (\%)

\begin{tabular}{lccc}
\hline 运输方式 & $2017-2020$ 年 & $2021-2025$ 年 & 2026-2030年 \\
\hline 铁路 & $1.50 \sim 1.90$ & $-0.90 \sim-1.10$ & $-2.80 \sim-3.10$ \\
公路及航空 & $2.10 \sim 2.30$ & $-0.80 \sim-1.10$ & $-9.40 \sim-9.60$ \\
水路 & $-1.45 \sim-1.55$ & $-2.30 \sim-2.40$ & $-3.32 \sim-3.52$ \\
管道 & $1.70 \sim 2.10$ & $-0.70 \sim-1.10$ & $-3.60 \sim-4.10$ \\
\hline
\end{tabular}

\section{2 建议}

根据以上研究, 本文提出如下建议:

（1）优化交通运输结构,加快构建节能低碳的 综合交通运输体系

为了降低运输碳强度, 遵照最优原则, 充分发 挥各交通运输方式的优势与组合效率, 选择最适宜 的运输方式, 由于本文研究结果表明水路及铁路运 输达峰时间较早, 因此应加大发展铁路及水运等低 碳运输方式的力度。加快发展公交、地铁和轻轨等 大容量公共交通,大力推崇自行车、步行等慢行交 通,提高绿色出行的比例, 积极开展 “绿色交通都 市”的示范工程, 提升东北三省交通运输系统的低 碳化水平。

(2)提高运输效率, 大力发展低碳能源

提高运输效率是减少能源使用量的基础, 是降 低运输碳强度的重要手段,也是实现交通运输低碳 发展的重要途径。如合理规划铁路运行线路及座 位与卧铺比例, 选择适宜的高铁站点及中转站点, 提高运输效率; 在公路运输方面, 大力发展直达运 输, 合理配置车型, 在不超载的前提下提高运输车 辆的实载率降低空载率; 在水路运输方面,减少输 送或装卸机械的空转时间, 提高装卸生产效率。本 文研究结果显示技术突破情景达峰时间较早,因此 要大力开发替代能源、可再生能源及清洁能源, 加 快交通运输用能的低碳转型。积极落实“气化交 通”的发展战略, 着力推广天然气车辆及船舶, 大力 推进铁路运输的电气化改造, 深人开展港口装卸机 械“油改电”的工作。

(3)加强低碳创新, 提高东北三省交通运输的 低碳能力 
坚持创新驱动战略,提高投资效率并降低投资 碳强度,促进资源节约循环高效利用, 提升科技创 新对东北三省低碳运输发展的驱动力和支撑力。 加强适用低碳技术的相关产品的研发与推广, 如飞 机、高铁、运输船舶、混合动力汽车、燃料电池汽车 及电动汽车等运载工具。

\section{参考文献(References):}

[1 ] 刘淑乔. 《全球碳预算报告》:2017年全球碳排放强劲反弹[EB/ OL]. (2017- 11- 22) [2018- 12- 25]. http://www.cma.gov.cn/ 2011xwzx/2011xqhbh/2011xdtxx/201711/t20171122_456610.ht ml. [Liu S Q. 2017 Global Carbon Budget Report: 2017 Global Carbon Emissions Rebounded Strongly[EB/OL]. (2017- 11-22) [2018- 12- 25]. http://www.cma.gov.cn/2011xwzx/2011xqhbh/ 2011xdtxx/201711/t20171122_456610.html.]

[2] 世界自然基金会. 2013 年在华非化石能源企业碳排放强度排 行榜报告 [EB/OL]. (2013-12) [2018- 12-25]. https://max. book118.com/html/2018/0907/6003200032001215.shtm. [World Wide Fund for Nature. Report on the Carbon Emission Intensity Ranking of Non-Fossil Energy Enterprises in China in 2013[EB/ OL]. (2013-12) [2018-12- 25]. https://max.book118.com/html/ 2018/0907/6003200032001215.shtm.]

[3] 喻洁, 达亚涁, 欧阳斌. 基于LMDI 分解方法的中国交通运输行 业碳排放变化分析[J]. 中国公路学报, 2015, 28(10): 112-119. [Yu J, Da Y B, Ouyang B. Analysis of carbon emission changes in China's transportation industry based on LMDI decomposition method[J]. China Journal of Highway and Transport, 2015, 28(10): 112-119.]

[4] 王泽宇, 徐静, 王炎熙. 中国海洋资源消耗强度因素分解与时空 差异分析[J]. 资源科学, 2019, 41(2): 301-312. [Wang Z Y, Xu J, Wang Y X. Factor decomposition and spatio-temporal difference analysis in marine resource consumption intensity in China[J]. Resources Science, 2019, 41(2): 301-312.]

[5]胡振, 何晶晶, 王玥. 基于 IPAT-LMDI 扩展模型的日本家庭碳 排放因素分析及启示 [J]. 资源科学, 2018, 40(9): 1831-1842. [Hu Z, He J J, Wang Y. Factor analysis and enlightenment of household carbon emissions in Japan based on IPAT-LMDI extension model[J]. Resources Science, 2018, 40(9): 1831-1842.]

[6] 谢守红, 蔡海亚, 夏刚祥. 中国交通运输业碳排放的测算及影响 因素[J]. 干旱区资源与环境, 2016, 30(5): 13-18. [Xie S H, Cai H Y, Xia G X. Calculation of the carbon emissions of Chinese transportation industry and the driving factors[J]. Journal of Arid Land Resources and Environment, 2016, 30(5): 13-18.]

[7] 朱勤, 彭希哲, 陆志明, 等. 中国能源消费碳排放变化的因素分
解及实证分析[J]. 资源科学, 2009, 31(12): 2072-2079. [Zhu Q, Peng X Z, Lu Z M, et al. Factors decomposition and empirical analysis of variations in energy carbon emission in China[J]. Resources Science, 2009, 31(12): 2072-2079.]

[8] 吕倩. 京津冀地区汽车运输碳排放影响因素研究[J]. 中国环境 科学, 2018, 38(10): 3689-3697. [Lv Q. Study on the driving factors of vehicle transport carbon emissions in Beijing-Tianjin-Hebei region[J]. China Environmental Science, 2018, 38(10): 36893697.

[9]吴青龙, 王建明, 郭不斌. 开放 STIRPAT 模型的区域碳排放峰 值研究: 以能源生产区域山西省为例[J]. 资源科学, 2018, 40 (5): 1051-1062. [Wu Q L, Wang J M, Guo P B. Peak regional carbon emissions based on open STIRPAT modeling in an energy: Producing region of Shanxi[J]. Resources Science, 2018, 40(5): 1051-1062.]

[10] 陈占明, 吴施美, 马文博, 等. 中国地级以上城市二氧化碳排放 的影响因素分析: 基于扩展的 STIRPAT 模型[J]. 中国人口·资 源与环境, 2018, 28(10): 45-54. [Chen Z M, Wu S M, Ma W B, et al. Driving forces of carbon dioxide emission for China's cities: Empirical analysis based on extended STIRPAT Model[J]. China Population, Resources and Environment, 2018, 28(10): 45-54.]

[11] 刘满芝, 刘贤贤. 基于 STIRPAT 模型的中国城镇生活能源消费 影响因素研究[J]. 长江流域资源与环境, 2017, 26(8): 11111122. [Liu M Z, Liu X X. Research on the factors affecting urban domestic energy consumption based on STIRPAT model[J]. Resources and Environment in the Yangtze Basin, 2017, 26(8): 1111-1122.]

[12] Vaninsky A. A factorial decomposition of $\mathrm{CO}_{2}$ emissions: A generalized Divisia index approach[J]. Energy Economics, 2014, 45: 389-400.

[13] Ma H T, Sun W, Wang S J, et al. Structural contribution and scenario simulation of highway passenger transit carbon emissions in the Beijing-Tianjin-Hebei metropolitan region, China[J]. Resources, Conservation \& Recycling, 2019, 140(2): 209-215.

[14] 段福梅. 中国二氧化碳排放峰值的情景预测及达峰特征: 基于 粒子群优化算法的 BP 神经网络分析[J]. 东北财经大学学报, 2018, (5): 19-27. [Duan F M. Scenario prognostics and characteristics of China's carbon dioxide emissions peak: A BP neural network analysis based on particle swarm optimization[J]. Journal of Dongbei University of Finance and Economics, 2018, (5): 19-27.]

[15] 刘俊伶, 孙一赫, 王克, 等. 中国交通部门中长期低碳发展路径 研究[J]. 气候变化研究进展, 2018, 14(5): 513-521. [Liu J L, Sun Y H, Wang K, et al. Study on mid-and long-term low carbon development pathway for China's transport sector[J]. Climate Change Research, 2018, 14(5): 513-521.]

[16] 袁长伟, 李若影, 芮晓丽, 等. 陕西省交通运输业碳排放影响因 素分解研究[J]. 长安大学学报(社会科学版), 2016, 18(2): 38- 
42. [Yuan C W, Li R Y, Rui X L, et al. Decomposition of the impact factors of carbon emission in Shaanxi transportation industry [J]. Journal of Chang' an University (Social Science Edition), 2016, 18(2): 38-42.]

[17] 360 百科. 换算周转量[EB/OL]. (2019-01-22) [2019-04-22]. https: //baike. so. com/doc/7872813-8146908. html. [360 Encyclopedia. Conversion Turnover[EB/OL]. (2019-01-22) [2019-0422]. https: //baike. so. com/doc/7872813-8146908. html.]

[18] 国家发改委能源研究所. 中国省级温室气体清单编制指南[EB/ OL]. (2011-05) [2018-12-25]. https: //wenku. baidu. com/view/ 7ae95325f111f18583d05a67. html. [National Development and Reform Commission Energy Research Institute. China's Provincial Greenhouse Gas Inventory Compilation Guide[EB/OL]. (201105) [2018-12-25]. https: //wenku. baidu. com/view/7ae95325f1 11f18583d05a67. html.]

[19] 辽宁省统计局. 辽宁省统计年鉴(2005-2017)[M]. 北京: 中国统 计出版社, 2006-2018. [Liaoning Provincial Bureau of Statistics. Liaoning Province Statistical Yearbook 2005-2017[M]. Beijing: China Statistics Press, 2005-2017.]

[20] 吉林省统计局. 吉林省统计年鉴(2005-2017)[M]. 北京: 中国统 计出版社, 2006-2018. [Jilin Provincial Bureau of Statistics. Jilin Province Statistical Yearbook 2005-2017[M]. Beijing: China Statistics Press, 2005-2017.]

[21] 黑龙江省统计局. 黑龙江省统计年鉴(2005-2017)[M]. 北京: 中 国统计出版社, 2006-2018. [Heilongjiang Provincial Bureau of Statistics. Heilongjiang Province Statistical Yearbook 2005-2017 [M]. Beijing: China Statistics Press, 2005-2017.]

[22] 国家统计局. 中国能源统计年鉴(2005-2017)[M]. 北京: 中国统 计出版社, 2006-2018. [National Bureau of Statistics. China Energy Statistics Yearbook 2005-2017[M]. Beijing: China Statistics Press, 2006-2018.]

[23] Lin B, Ouyang X. Analysis of energy-related $\mathrm{CO}_{2}$ (Carbon Dioxide) emissions and reduction potential in the Chinese non-metallic mineral products industry[J]. Energy, 2014, 68: 688-697.

[24] 国务院. “十三五”现代综合交通运输体系发展规划[EB/OL]. (2017-02-03) [2018-12-25]. http: //www. mot. gov. cn/zhuanti/ shisanwujtysfzgh/guihuawenjian/201703/t20170301_2170528. html. [The State Council of the People's Republic of China. "Thirteenth Five-Year" Modern Comprehensive Transportation System Development[EB/OL]. (2017-02-03) [2018-12-25]. http: //www. mot. gov. cn/zhuanti/shisanwujtysfzgh/guihuawenjian/201703/ t20170301_2170528. html.]

[25] 邢丽敏. 中国交通能耗影响因素及节能减排潜力分析[D]. 西 安: 陕西师范大学, 2017. [Xing L M. Analysis of Factors Affecting Traffic Energy Consumption in China and Energy Saving and Emission Reduction Potential[D]. Xi' an: Shaanxi Normal University, 2017.]
[26] 能源局. 能源局发布《能源发展“十三五”规划》[EB/OL]. (201701-05) [2018-12-25]. http: //www. gov. cn/xinwen/2017-01/05/ content_5156795. htm\#1. [National Energy Administration. The Energy Bureau Released the "13th Five-Year Plan for Energy Development”[EB/OL]. (2017-01-05) [2018-12-25]. http: //www. gov. cn/xinwen/2017-01/05/content_5156795. htm\#1.]

[27] 国务院. 国务院办公厅印发《能源发展战略行动计划(20142020 年) $\rangle[J]$. 建设科技, 2014, 12(22): 6. [The State Council of the People's Republic of China. The General Office of the State Council issued the "energy development strategic action plan (20142020)"[J]. Construction Technology, 2014, 12(22): 6.]

[28] 林伯强, 刘希颖. 中国城市化阶段的碳排放: 影响因素和减排策 略[J]. 经济研究, 2010, 45(8): 66-78. [Lin B Q, Liu X Y. China's carbon dioxide emissions under the urbanization process: Influence factors and abatement policies[J]. Economic Research Journal, 2010, 45(8): 66-78.]

[29] 国家发改委, 能源局. 能源生产和消费革命战略(2016-2030) [J]. 电器工业, 2017, 17(5): 39-47. [National Development and Reform Commission, National Energy Administration. Energy production and consumption revolution strategy (2016-2030)[J]. China Electrical Equipment Industry, 2017, 17(5): 39-47.]

[30] 网易新闻. 中科院预测: 2016年固定资产投资增速大约为 $10 \%$ 左右[EB/OL]. (2016-01-05) [2018-12-25]. http: //news. 10jqka. com. cn/20160105/c586875924. shtml. [Netease News. The Chinese Academy of Sciences Predicts That the Growth Rate of Fixed Asset Investment in 2016 Will Be about 10\%[EB/OL]. (2016-0105) [2018- 12- 25]. http://news. 10jqka. com. cn/20160105/ c586875924. shtml.]

[31] 中华人民共和国交通部. 交通运输节能环保“十三五”规划[EB/ OL]. (2016- 05-31) [2018-12-25]. https: //wenku. baidu. com/ view/322b47029b89680202d82592. html. [Ministry of Transport of the People's Republic of China. Traffic Energy Conservation and Environmental Protection "13th Five- Year Plan" [EB/OL]. (2016-05-31) [2018-12-25]. https: //wenku. baidu. com/view/ 322b47029b89680202d82592. html.]

[32] Chen S Y, Santos-Paulino A U. Energy consumption restricted productivity re-estimates and industrial sustainability analysis in post-reform China[J]. Energy Policy, 2013, 57: 52-60.

[33] Ramírez A, Keizer C D, Sluijs J P V D, et al. Monte Carlo analysis of uncertainties in the Netherlands greenhouse gas emission inventory for 1990-2004[J]. Atmospheric Environment, 2008, 42(35): $8263-8272$.

[34] 新华社. “十二五”规划纲要[EB/OL]. (2011-03-16) [2018-1225]. http://www.china.com.cn/policy/txt/2011- 03/16/content_ 22156007.htm. [Xinhua News Agency. Twelfth Five- Year Plan [EB/OL]. (2011-03-16) [2018-12-25]. http://www.china.com.cn/ policy/txt/2011-03/16/content_22156007.htm.] 


\title{
Empirical decomposition and forecast of peak carbon emissions of five major transportation modes :
} Taking the three provinces in Northeast China as examples

\author{
WANG Yong ${ }^{1,2}$, HAN Shuwan ${ }^{1}$, LI Jiayuan ${ }^{3}$, LI Bo ${ }^{1}$ \\ (1. School of Statistics, Dongbei University of Finance and Economics, Dalian 116025, China; \\ 2. Postdoctoral Research Station, Dongbei University of Finance and Economics, Dalian 116025, China; \\ 3. School of Marine Engineering, Dalian Maritime University, Dalian 116026, China)
}

\begin{abstract}
The transportation industry is one of the key industries necessary for the development of the national economy and the everyday life of residents, and it is also one of the main sources of carbon emissions. High energy consumption and high pollution have always been problems in the transportation industry. The effective control of carbon emissions in the transportation industry is greatly important for achieving China's carbon emission peak target. This study took the three provinces in Northeast China as the research object and conducted a detailed examination on the carbon emissions of five different modes of transportation: road, railway, air, waterway, and pipeline transportation. First, we used the generalized divisia index method (GDIM) to examine the factors affecting the carbon emissions of the five transportation modes from 2005 to 2016 and Monte Carlo simulation to calculate the carbon emissions of the five major transportation modes in 2017-2030. The annual average rate of change was used for dynamic scenario analysis. The results show that the scale of investment is the primary factor affecting the carbon emissions of railway, road, air, and pipeline transportation. The transportation scale is the primary factor affecting the carbon emissions of waterway transportation. During the same period, the influencing factors are different. In different time periods, the same factors also affect growth or reduction of carbon emissions differently. Except under the baseline scenario, the carbon emissions of the five modes of transportation in 2017-2030 will gradually decline; the carbon emissions of the five types of transportation are expected to decline the most under the technological breakthrough scenario. The development of transportation equipment using clean energy, performance improvement, and vigorous promotion should be the main development path for energy conservation and emission reduction in the future transportation industry.
\end{abstract}

Key words: transportation industry; carbon peak; empirical decomposition; scenario simulation; Monte Carlo simulation; three provinces in Northeast China 